\title{
Correction to: Stress-induced changes in body surface temperature are repeatable, but do not differ between urban and rural birds
}

\author{
Joshua K. R. Tabh ${ }^{1,2}$ (D) Gabriela F. Mastromonaco ${ }^{2}$. Gary Burness ${ }^{3}$
}

Published online: 4 March 2022

(c) Springer-Verlag GmbH Germany, part of Springer Nature 2022

\section{Correction to: Oecologia \\ https://doi.org/10.1007/s00442-022-05120-z}

Authors would like to correct the incorrect placement of subsections under the Material and Methods heading in the original publication.

The original article has been corrected.

The original article can be found online at https://doi.org/10.1007/ s00442-022-05120-z.

Joshua K. R. Tabh

joshua.k.tabh@gmail.com

1 Environmental and Life Sciences Graduate Program, Trent University, Peterborough, ON K9L 0G2, Canada

2 Department of Wildlife and Science, Toronto Zoo, Scarborough, ON M1B 5K7, Canada

3 Department of Biology, Trent University, Peterborough, ON K9L 0G2, Canada 